\title{
Distribuição das espécies do gênero Loxosceles Heinecken \& Lowe, 1835 (Araneae; Sicariidae) no Estado do Paraná
}

\author{
Loxosceles Heinecken \& Lowe, 1835 (Araneae; Sicariidae) \\ species distribution in the State of Paraná
}

\author{
Emanuel Marques-da-Silva ${ }^{1}$ e Marta Luciane Fischer $^{2}$
}

\begin{abstract}
RESUM0
0 Estado do Paraná registra anualmente uma média de 2.577 acidentes loxoscélicos por ano, logo o conhecimento da distribuição das espécies do gênero Loxosceles é extremamente importante para elaboração de ações de controle e manejo. Realizou-se o mapeamento das Loxosceles tombadas em diferentes coleções científicas. Foram registradas 1.561 aranhas, identificadas como Loxosceles intermedia (67\%), Loxosceles gaucho (19,5\%), Loxosceles laeta (10,8\%) e Loxosceles hirsuta $(2,4 \%)$, provenientes de 20 regionais de saúde e 69 municípios. Loxosceles intermedia ocorreu em todas as regiões do estado ( 50 municípios), enquanto Loxosceles gaucho ocorreu no norte e noroeste, ( 17 municípios) , Loxosceles laeta no sul (13 municípios) e Loxosceles hirsuta nas regiões oeste e centro (10 municípios). № Paraná ocorrem quatro das oito espécies de Loxosceles registradas no Brasil. Estudos nos locais de incidência e levantamentos em áreas ainda não amostradas devem ser realizados, devido à importância médica que os acidentes causados por essas aranhas têm produzido.
\end{abstract}

Palavras-chaves: Distribuição geográfica. Loxoscelismo. Loxosceles. Picada de aranha.

\begin{abstract}
The State of Paraná registers on average 2,577 loxoscelic accidents annually. For the elaboration of control and management programs one should first determine the distribution of the species of the genus Loxosceles. A mapping was performed of Loxosceles references in various scientific collections. Atotal of 1,561 spiders were found, identified as Loxosceles intermedia (67\%), Loxosceles gaucho (19.5\%), Loxosceles laeta (10.8\%) and Loxosceles hirsuta (2.4\%), originating from 20 regional and 69 municipal health districts. Loxosceles intermedia was present in all areas of the state ( 50 municipal districts), while Loxosceles gaucho occurred in the north and northwest, (17 municipal districts), Loxosceles laeta in the south (13 municipal districts) and Loxosceles hirsuta in the west and central areas (10 municipal districts). Paraná has four of the eight species of Loxosceles registered in Brazil. Given the medical importance of accidents caused by these spiders, it is necessary to perform studies on the location of such incidents and investigate areas that have not yet been sampled.
\end{abstract}

Key-words: Geographic distribution. Loxoscelism. Loxosceles. Spider bites.

№ Brasil, de 1990 a 1993, foram registrados 17.785 acidentes araneídicos, a maioria nas Regiões Sul e Sudeste ${ }^{10}$. Daqueles notificados de 1988 a 2001 $(\mathrm{n}=61.775)$, o Estado do Paraná representou $44 \%(n=27.364)$ do total e $72 \%$ da Região Sul $(\mathrm{n}=38.133)^{10}$. 0 Paraná é 0 estado brasileiro que notifica 0 maior número de acidentes por aranhas Loxosceles no país, sendo 62,2\% (595) em 1988-1989 e 77,4\% (923) em 1989-199016.

A maior casuística sobre loxoscelismo estudada até 0 momento foi apresentada por Marques-da-Silva ${ }^{13}$ sendo analisados 20.620 casos de acidentes registrados no Paraná em apenas oito anos (1993 a 2000), distribuídos em 43,9\% ( $\mathrm{n}=175)$ dos municípios.

0 gênero Loxosceles é cosmopolita e possui mais de 100 espécies que têm como centro de origem a África e as Américas ${ }^{11}$. Destas espécies, 20 são endêmicas da África, 50 da América do Norte e Central e 30 da América do Sul ${ }^{11}$. Para uma melhor sistematização das espécies, 0 autor dividiu-as em grupos levando em consideração caracteres da genitália de machos e fêmeas. Os grupos presentes no continente americano são: reclusa, na América do Norte e Central e gaucho, spadicea, amazônica e

\footnotetext{
1. Seção de Animais Peçonhentos do Centro de Produção e Pesquisa de Imunobiológicos. 2. Núcleo de Estudos do Comportamento Animal Departamento de Biologia do Centro de Ciências Biológicas e da Saúde da Pontifícia Universidade Católica do Paraná.

Endereço para correspondência: Dr. Emanuel Marques da Silva. R. Targino da Silva, s/no, 83.302-160 Piraquara, PR.

Tel: $55413673-8825$

e-mail: ems@pr.gov.br

Recebido para publicação em 7/6/2004

Aceito em 3/5/2005
} 
laeta, na América do Sul. Duas espécies detêm 0 status de cosmopolitas: L laeta ( Nicolet, 1849) originária da América do Sul e L rufescens ( Dufour, 1820) da Europa, sendo amplamente distribuídas devido ao transporte passivo através do comércio ${ }^{11}$.

№ Brasil ocorrem oito espécies de Loxosceles, sendo quatro endêmicas do país: L similis Moenkhaus, 1998 ( PA, SP, MG e MS) , L gaucho Gerstch, 1967 ( RS e SP) , L amazonica Gerstch, 1967 (AM, MG e MA) e L puortoi Martins, Knysak \& Bertani, 2002 (T0) e quatro ocorrendo também em países vizinhos: L laeta (RS, SP, RJ, MG e PR) ; L intermedia Mello-Leitão, 1934 (DF, RJ, SP e RS) ; L hirsuta Mello-Leitão, 1931 (RS e PR) e L adelaida Gerstch, 1967 (RJ) ${ }^{2} 11$.

A taxonomia do grupo foi alvo de inúmeros debates, mais intensamente na década de 1960 principalmente com respeito ao número e sinonímias das espécies de Loxosceles ocorrentes na América do Sul, as quais tiveram a última revisão feita por Gerstch $^{11}$, com 19 espécies novas e mapas de distribuição. № entanto esses dados devem ser avaliados com cautela, pois 0 autor não revisou pessoalmente todos os animais, valendo-se do registro do livro tombo de coleções científicas. Segundo 0 autor, o problema da sistemática de Loxosceles relaciona-se com a sua natureza haplógina, em que há uniformidade nos caracteres somáticos especialmente da genitália, além das grandes semelhanças entre as espécies devido a uma explosiva radiação adaptativa que produziu espécies muito próximas, provavelmente por explorarem hábitos e habitats semelhantes.

Apesar do Paraná ser 0 estado com maior número de casos de loxoscelismo, pouco se sabe sobre a distribuição das espécies. Para 0 estado, os dados disponíveis datam da metade do século passad $0^{15}$ e mais recentemente Fischer ${ }^{4}$ realizou levantamento das espécies ocorrentes no município de Curitiba. 0 presente estudo teve como objetivo realizar um mapeamento da distribuição das espécies do gênero Loxosceles no Estado do Paraná.

\section{MATERIAL E MÉTODOS}

0 Estado do Paraná possui $468 \mathrm{~km}$ de extensão na direção norte sul $\left(22^{\circ} 29^{\prime} 30^{\prime \prime}\right.$ e $\left.26^{\circ} 42^{\prime} 59^{\prime \prime}\right)$ e $674 \mathrm{~km}$ de leste a oeste ( 4802'24" e 5437'38") ${ }^{12}$, distribuídos em 399 municípios que são divididos administrativamente em 22 regionais de saúde (RS) .

A distribuição parcial das espécies do gênero Loxosceles no Estado do Paraná foi obtida com base nos registros em coleções científicas. Para tal foram analisadas todos os exemplares originários do Paraná tombados nas seguintes coleções: Coleção Aracnológica Vera Regina von Eickstedt da Seção de Animais Peçonhentos do Centro de Produção e Pesquisa de Imunobiológicos (CPPI)/Secretaria de Estado da Saúde do Paraná (SESA), Coleção Rudolf Bruno Lange do Museu de História Natural Capão da Imbuia Secretaria Municipal de Meio Ambiente/Prefeitura Municipal de Curitiba, coleção do Laboratório de Artrópodes do Instituto Butantan, SP, coleção de aranhas no Museu Nacional da Universidade Federal do Rio de Janeiro, RJ e Museu de Zoologia da Universidade de São Paulo, SP. Destas coleções, apenas a presente no CPPI é voltada para a recepção e identificação principalmente de animais de interesse médico, encaminhados pelas Regionais de Saúde - RS de todo 0 estado. Nesta coleção também são depositados exemplares "testemunhas" de coletas de aranhas para extração de veneno para produção de soro anti-veneno (soro antiloxoscélico). Os mapas foram gerados através do programa TABWIN (v.2.0).

\section{RESULTADOS E DISCUSSÃ0}

A distribuição do gênero Loxosceles no Estado do Paraná englobou 20 regionais de saúde e 69 municípios. A coleção científica que melhor representou a ocorrência do gênero para o Estado do Paraná foi a presente no CPPI, constando com uma amostragem histórica de 12 anos (1992 a 2003) e fornecendo 0 registro de 1.458 aranhas. As demais coleções trazem dados pontuais com poucos registros para o Estado do Paraná.

No Paraná estão presentes quatro das oito espécies de Loxosceles descritas para 0 Brasil: L. intermedia, L laeta, L gaucho e L hirsuta.

A espécie com maior percentual de exemplares 1.051 $(65,1 \%)$ nas coleções consultadas foi $L$ intermedia. Foi também a espécie que apresentou a distribuição mais ampla pelo estado, tendo sido encontrada em todas as regiões do Estado abrangendo 50 municípios agrupados em 13 regionais de saúde (Figura 1A). L intermedia, cujo holótipo é de Petrópolis RJ, faz parte do grupo spadicea junto com L hirsuta e L spadicea ${ }^{11}$. Embora a espécie apresente predomínio no sul do país (Paranát; Rio Grande do Sul2; Santa Catarina ${ }^{18}$ ), sua ocorrência é registrada também em estados do sudeste (Rio de Janeiro, Minas Gerais) e do centro oeste (Brasília) ${ }^{11}$. A biologia, ecologia e comportamento da espécie foram amplamente estudados por Fischer ${ }^{56}$ e Fischer e Vasconcellos-Neto ${ }^{9}$. Os autores, comparando os padrões apresentados por L intermedia e L laeta, espécies ocorrentes no município de Curitiba, chegaram a conclusão de que L intermedia possui hábitos mais generalistas, se locomove mais e tolera mais o filhote coespecífico do que L laeta. Fatores estes, que favorecem a sua instalação e proliferação em diferentes substratos e ambientes. Os dados do presente estudo corroboram com os hábitos generalistas da espécie, uma vez que a sua ocorrência abrange áreas com temperatura, umidade relativa do ar e altitudes contrastantes ${ }^{12}$.

Aespécie L gaucho foi a segunda espécie melhor amostrada 304 (19,5\%). A espécie predominou nos municípios do norte e noroeste do Estado, principalmente fronteira com São Paulo e Mato Grosso do Sul, sendo distribuída por 17 municípios em sete RS ( Figura 1B) . 0 grupo gaucho é composto ${ }^{11}$ por L gaucho, L similise L adelaida e L amazônica, espécie única do grupo amazônica se constitui de uma forma isolada do grupo gaucho. L gaucho é uma espécie endêmica do Brasil e predomina nos estados do Sudeste, sendo o holótipo proveniente de São Paul ${ }^{11}$. Neste estado, L gaucho é a espécie predominante nos locais de acidentes ${ }^{3}$. A biologia da espécie foi estudada por Rinaldi cols ${ }^{17}$, Buckup $^{2}$ não confirmou a ocorrência da espécie no Rio Grande do Sul como havia sido referida por Gersth ${ }^{11}$. Para o Paraná, 
Fischer ${ }^{4}$ localizou um foco isolado de L gaucho em um eucalipto presente em uma área de camping em um clube de campo.

A espécie L laeta foi representada por 10,8 \% ( $\mathrm{n}=169)$ dos registros, ocorrendo 13 municípios e 7 regionais de saúde (Figura 1C). L laeta predominou nos municípios localizados na região sul do estado. Esta espécie é nativa do sul da América Latina provavelmente Argentina ou Chile, devido a inúmeros problemas de sinonímia são encontrados tipos no Peru, Chile e Argentina. Atualmente, a espécie ocorre em inúmeros países distribuídos por todos os continentes ${ }^{11}$. A alta capacidade de viver um longo período em inanição é um fator que favorece a essa espécie estabelecer populações em diferentes locais onde é introduzida acidentalmente. Porém, Fischer ${ }^{6}$ chama a atenção para a pequena dispersão de $L$ laeta a qual estabeleceu focos apenas ao redor do ponto de introdução. 0 grupo laeta é grande e representado por mais de 24 espécies, sendo a maioria $(n=15)$ endêmica do Peru ${ }^{11}$. A espécie mais recentemente descrita para o Brasil (L puortoi) faz parte do grupo laeta ${ }^{14}$.

Aespécie L hirsuta foi representada apenas por 37 indivíduos e apesar de se distribuir em diferentes regiões do Estado, ocorreu apenas em 10 municípios e nove RS (Figura 1D) . L hirsuta, cujo holótipo é de Cacimbinhas RS, foi erroneamente incluída na lista de distribuição para o Paraná ( Curitiba) ${ }^{15}$, uma vez que Fischer ${ }^{4}$ revisando o material tombado na coleção do Museu de História Natural Capão da Imbuía constatou se tratar de L laeta e L intermedia. Após esse registro, a espécie não foi mais notificada para 0 estado. Resultado semelhante foi obtido por Buckup ${ }^{2}$ para o Rio Grande do Sul, onde a ocorrência da espécie não foi confirmada. A partir de 1996 foram recebidos na coleção do CPPI dois machos, provenientes de Cascavel (1996/1998) e uma fêmea, proveniente de Telêmaco Borba (1998). Em outubro de 1998, 17 indivíduos ( 5 machos, 9 fêmeas e 3 jovens) foram coletados intra e peridomiciliarmente em edificações na Usina Hidrelétrica Julio de Mesquita Filho, no município de Cruzeiro do Iguaçu. Em junho de 1999, cinco indivíduos ( 3 machos, 1 fêmea e 1 jovem) foram coletados em um depósito e em entulhos, na vila de moradores da Usina Hidrelétrica de Salto Osório, município de Quedas do Iguaçu. Nestes mesmos ambientes foram também coletados exemplares L intermedia. Desta forma, esses registros confirmam a ocorrência de L hirsuta para 0 Estado do Paraná. 0 comportamento copulatório, a fertilidade e 0 desenvolvimento pós-embrionário da espécie foram estudados por Fischer \& Marques-da-Silva ${ }^{78}$. Os indivíduos de L hirsuta são superficialmente semelhantes aos de L. intermedia, principalmente no que diz respeito à tonalidade da coloração

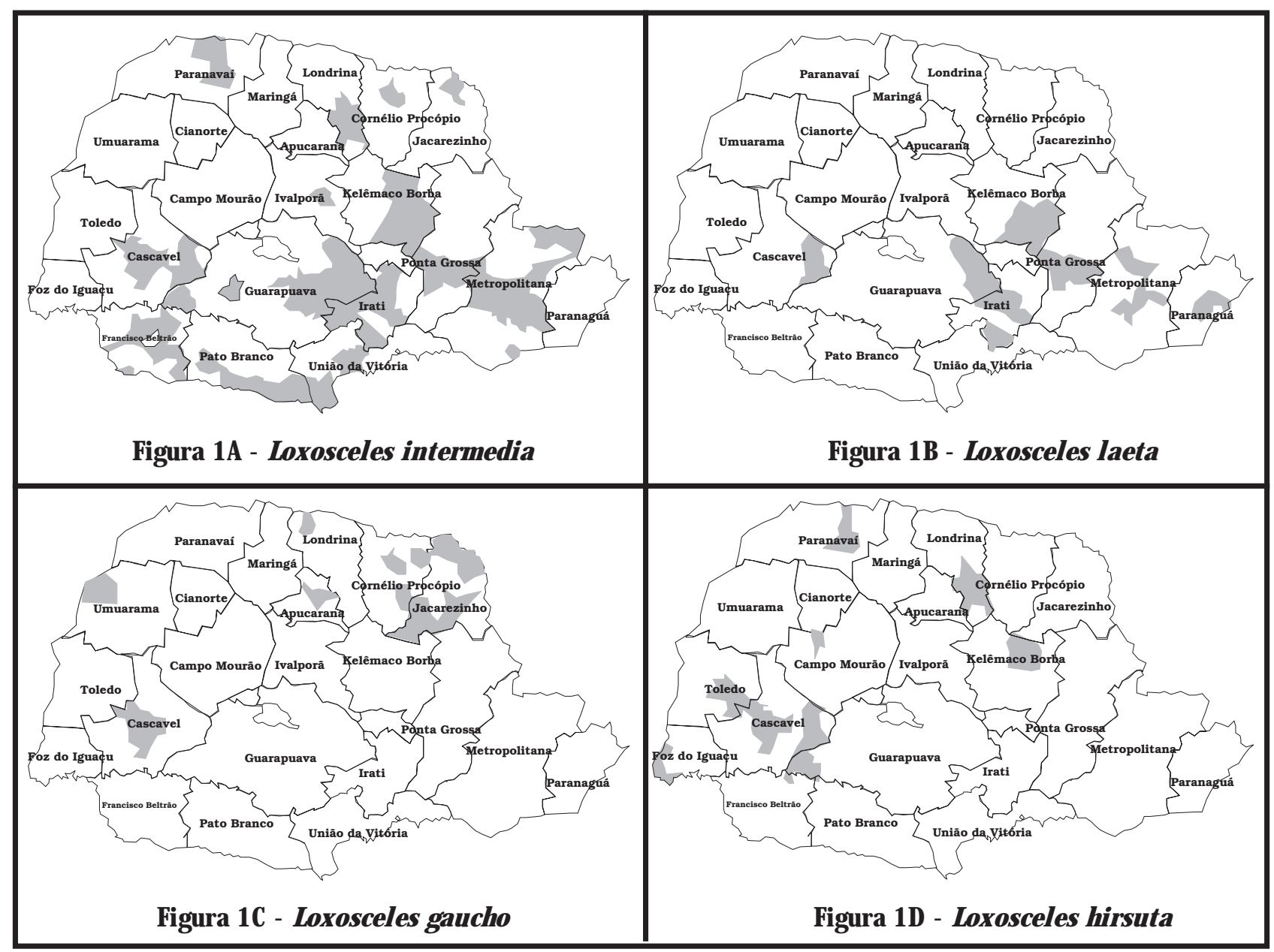

Figura 1 - Mapas da distribuição das espécies do gênero Loxosceles por municípios do Estado do Paraná - BR: Loxosceles intermedia (Figura 1A), Loxosceles laeta (Figura 1B), Loxosceles gaucho (Figura 1C) e Loxosceles hirsuta (Figura 1D). As divisas correspondem às vinte e duas Regionais de Saúde da SESA, PR. 
do corpo e provavelmente por fazerem parte do mesmo grupo spadicea. 0 menor tamanho de L hirsuta somada à delicadeza do tegumento do abdome o qual dificulta a preparação dos receptáculos seminais, são fatores que contribuem para que possam ser confundidas com jovens de L intermedia, e automaticamente serem subnotificadas em outras coleções. Gertsch ${ }^{11}$ sugere que L hirsuta e L. surata sejam expressões geográficas de uma mesma espécie.

Os estudos de Fischer ${ }^{6}$ mostram diferenças relevantes entre 0 hábito de L intermedia e L laeta, os quais foram utilizados pela autora para explicar o predomínio de L intermedia no município de Curitiba. Na capital, L laeta ocorre principalmente no intradomicílio de construções antigas ${ }^{6}$.

De maneira semelhante, em outras localidades, como a região de Lauro Müller - SC, a espécie ocorre associada a antigas construções da área rural onde estabelece grandes populações (E Marques-da-Silva: comunicação pessoal, 2003). Já L intermedia é encontrada no intra-e no peridomicílio, tanto em Curitiba ${ }^{6}$ quanto nos demais municípios do estado (E Marquesda-Silva: comunicação pessoal, 2003). A menor resistência de L laeta às altas temperaturas ${ }^{9}$ corroboram com os dados do presente estudo, em que ocorrências pontuais de L laeta são confrontadas com a distribuição mais ampla de L intermedia, englobando áreas de ocorrência de temperaturas mais altas. 0 predomínio de L gaucho no norte do estado, sugere que a espécie seja mais adaptada a características climáticas especíicas da região, como altas temperaturas e pouca umidade ${ }^{12}$. Nessas condições, as aranhas são freqüentemente encontradas entre frestas de terras em barrancos, sob telhas e restos de entulhos de construção (E Marques-da-Silva: comunicação pessoal, 2003), onde provavelmente a temperatura é mais amena e a umidade maior, pois a ocorrência intradomiciliar é raramente registrada. Já L. hirsuta, parece representar pequenas populações distribuídas em focos dispersos pelo estado. A semelhança morfológica com L intermedia sugere avaliações mais cuidadosas na identificação desses exemplares.

Concluindo, no Estado Paraná ocorre metade das espécies de Loxosceles registradas para o Brasil. A distribuição dessas espécies evidencia a existência de padrões específicos relacionados a hábitos diferenciais

Os dados do presente estudo fornecem um panorama da distribuição do gênero pelo estado, ressaltando as características peculiares de cada espécie. Os recentes registros de loxoscelismo levam ao questionamento sobre quais teriam sido os fatores que favoreceram as espécies a ocuparem 0 ambiente antrópico. Infelizmente os questionamentos para a compreensão desse fenômeno estão relacionados ao fato de que o reconhecimento das espécies coincide com 0 registro de acidentes e, automaticamente, com 0 seu status de animal de importância médica. Desta forma, o acontecimento histórico pode ser abordado apenas por meio de hipóteses. A mesma consideração foi feita por Gertsch ${ }^{11}$ para L reclusa nos Estados Unidos. 0 autor discutiu, ainda, a hipótese de que a ocupação do ambiente antrópico teria sido recente, e que, justamente a melhoria das condições ambientais, tais como, amenizando temperaturas extremas, favoreceram a ocupação desse novo habitat e possibilitou 0 transporte passivo das aranhas pelo homem. № entanto, apesar deste ponto de vista ser tentador o mesmo deve ser considerado com cautela. Aimportância médica das espécies e 0 problema de saúde pública gerado no estado do Paraná demandam que sejam realizados estudos específicos nos locais de incidência e levantamentos em áreas ainda não amostradas, já que o número de acidentes tem sido significativamente maior que 0 registro de aranhas em áreas urbanas ${ }^{13}$. 0 monitoramento das populações dessas espécies poderá servir como instrumento na aplicação de medidas profiláticas, prevenindo o loxoscelismo.

\section{AGRADECIMENTOS}

Os autores agradecem aos funcionários das regionais de saúde que enviaram 0 material para tombamento na coleção científica da Seção de Animais Peçonhentos do CPPI. Agradecemos aos curadores das coleções de aranhas consultadas: Ricardo Pinto da Rocha, Antônio Brescovit, Julio César Moura Leite e Adriano Kury. Os autores agradecem os funcionários da Seção: Milton, Joel, Eliseu pelas coletas e organização da coleção.

\section{REFERÊNCIAS BIBLIOGRÁFICAS}

1. Bücherl W. Aranhas do gênero Loxosceles e loxoscelismo na América. Ciência e Cultura 13:213-224, 1961.

2. Buckup EH. Variação interpopulacional dos receptáculos em aranhas do grupo spadicea do gênero Loxosceles Heinecken \& Lowe, 1832 (Araneae; Scytodidae). Hieringia ser Zoologia 55:137-147, 1980.

3. Cardoso JLC, França FO, Eickstedt VD, Borges I, Nogueira, MT. Loxoscelismo: estudo de 242 casos (1980-1984). Revista da Sociedade Brasileira de Toxicologia 1:58-60, 1988.

4. Fischer ML. Levantamento das espécies do gênero Loxosceles Heinecken \& Lowe, 1832, no município de Curitiba, PR. Estudos de Biologia 38:6786, 1994.

5. Fischer ML. Biologia e Ecologia de Loxosceles intermedia Mello-Leitão, 1934 (Araneae; Sicariidae) no Município de Curitiba, PR. Dissertação de mestrado, Universidade Federal do Paraná, Curitiba, PR, 1996.

6. Fischer ML. Utilização do Habitat por Loxosceles intermedia MelloLeitão,1.934 e L laeta ( Nicolet, 1849) no Município de Curitiba, PR. Uma Abordagem Experimental sobre Aspectos Ecológicos e Comportamentais. Tese de Doutorado, Universidade Federal do Paraná, Curitiba, PR, 2002.

7. Fischer ML, Marques-da-Silva E. Comportamento sexual de Loxosceles hirsuta Mello-Leitão, 1931 (Araneae; Sicariidae). Estudos de Biologia 47: 7-14, 2001a.

8. Fischer ML, Marques-da-Silva E. Oviposição e desenvolvimento de Loxosceles hirsuta Mello-Leitão, 1931 (Araneae; Sicariidae). Estudos de Biologia 47: 14-20, $2001 \mathrm{~b}$.

9. Fischer ML, Vasconcellos-Neto J. Determination of the maximum and minimum lethal temperatures ( LT50) for Loxosceles intermedia MelloLeitão, 1934 and L laeta (Nicolet, 1849) (Araneae, Sicariidae). Journal of Thermal Biology 28: 563-567, 2003.

10. Fundação Nacional de Saúde. Manual de Diagnóstico e Tratamento de Acidentes por Animais Peçonhentos. Ministério da Saúde, Brasília, 1998.

11. Gerstsh WJ. The spider genus Loxosceles in South America. Bulletin American Museum Natural History 136:121-182, 1967.

12. Maack R. Geografia física do Estado do Paraná, 2aedição Livraria José Olympio, Rio de Janeiro, 1981. 
13. Marques-da-Silva E. Loxoscelismo no Estado do Paraná: epidemiologia dos acidentes causados por aranhas Loxosceles no período de 1993 a 2000. Dissertação de mestrado da Escola Nacional de Saúde Pública, Fundação Oswaldo Cruz, Rio de Janeiro, 2002.

14. Martins R, Knysak I, Bertani R. A new species of Loxosceles of the laeta group from Brazil (Araneae; Sicariidae). Zootaxa 94:1-6, 2002.

15. Mello-Leitão C. Aranhas do Paraná e Santa Catarina das coleções do Museu Paranaense. Arquivos do Museu Paranaense 6: 231-304, 1947.

16. Ribeiro LA, Eickstedt VRV, Rubio GBG, Konolsaisen FZ, Handar, Entres M, Campos VAFP, Jorge MT. Epidemiologia do acidente por aranhas do gênero Loxosceles Heniecken \& Lowe no Estado do Paraná (Brasil). Memórias do Instituto Butantan 55:19-26, 1993.

17. Rinaldi IMP, Forti LC, Stropa AA. On the development of the brown spider Loxosceles gaucho Gertsch (Araneae; Sicariidae) the nympho-imaginal period. Revista Brasileira de Zoologia 14: 697-706, 1997.

18. Sezerino UM, Zannin M, Coelho LK, Gonçalves Jr, Grando M, Mattosinho SG, Cardoso JLC, Eickstedt VRD, França FOS, Barbaro KC, Fan HW. A clinical and epidemiological study of Loxosceles spider envenoming in Santa Catarina, Brazil. Transactions of the Royal Society of Tropical Medicine and Hygiene 92: 546-548, 1998. 